DOI: $10.17957 / \mathrm{IJAB} / 15.1728$

http://www.fspublishers.org

\title{
DNA Fingerprinting and Genetic Diversity Assessment of GM Cotton Genotypes for Protection of Plant Breeder Rights
}

\author{
Shakra Jamil, Rahil Shahzad*, Muhammad Zaffar Iqbal, Erum Yasmeen and Sajid Ur Rahman \\ Agricultural Biotechnology Research Institute, Ayub Agricultural Research Institute, Faisalabad, Punjab Pakistan \\ *For Correspondence: rahilshahzad91@gmail.com \\ Received: 3 November 2020; Accepted: 28 December 2020; Published 25 March 2021
}

\begin{abstract}
DNA fingerprinting is rapid, easy, and efficient method for discrimination, identification and characterization of various genotypes for protection of plant breeder's rights (PBRs). Present study was designed for DNA fingerprinting and genetic diversity assessment of 25 GM cotton genotypes (possessing CrylAc gene) using 297 SSR markers through conventional PCR and Polyacrylamide gel electrophoresis. Out of 297 SSR markers, 25 markers were not amplified, 28 were monomorphic and 244 were polymorphic. A total of 1537 alleles were amplified among which $1294(84.18 \%)$ were polymorphic. PIC value in our study ranged from 0.08 to 0.93 with an average of 0.73 . Unique allelic pattern was observed for nineteen genotypes whereas six genotypes were identified using two-step identification methods. The UPGMA dendrogram divided the genotypes into two distinct clusters. Cluster I was comprised of 20 genotypes whereas cluster II was comprised of four genotypes. MNH$1020 \mathrm{did}$ not obey any clustering and remained separated. The results of the structure analysis were complementary to cluster analysis and the population was divided into two subgroups. Our results evidenced narrow genetic base of the cotton genotypes cultivated in Punjab Pakistan due to use of common parents in the pedigree/parentage. Further, we proposed a core set of markers for future DNA fingerprinting and genetic diversity studies. The information generated in this study will be helpful in variety registration and subsequent protection under PBRs. Further our findings will be useful in selection of SSR markers for future studies which are focused on DNA fingerprinting and genetic diversity assessment. (C) 2021 Friends Science Publishers
\end{abstract}

Keywords: Cluster analysis; Plant breeder rights; Polymorphic information content; Structure analysis

\section{Introduction}

Cotton (Gossypium spp.) also known as "White gold" is one of the major cash crops around the globe which is mainly cultivated to produce raw fiber for the textile industry (Singh 2017; Rehman et al. 2019; Jans et al. 2020). World fiber production equaled approximately 110 million metric tons in 2018, including 32 million tons of natural fibers and 79 million tons of chemical fibers. Cotton accounted for $80 \%$ of natural fiber production by weight (Townsend 2020) which shows its significance in international economies. Pakistan is the fourth largest lint producer of cotton (Shuli et al. 2018; Lalwani 2020).

Distinctness, uniformity, and stability (DUS) testing remain the sole scientific criteria for the protection and registration of new varieties in past (Pourabed et al. 2015). Earlier morphological and biochemical markers were used for DUS testing. The use of these markers produces inconsistent results because morphological and biochemical markers are influenced by the plant age, the environment and other factors. With the availability of molecular markers, it became possible to conduct rapid and accurate identification at the DNA level without the impact of environmental factors (Iqbal et al. 2017; Santhy et al. 2019). DNA fingerprinting is the rapid, easy, and most common method to discriminate, identify and characterize various cultivars to protect PBRs and promote marker-assisted breeding (Kalia et al. 2011). The technique has been revolutionized since the past three decades to distinguish the DNA polymorphism, biological identification, and documentation of species. Genetic profiling recapitulates the biological determination of species as well as traceability of diverse crop samples using the short tandem repeats. Through this PCR based approach, individual plant hybrids/varieties can be identified by acquiring a specific pattern of genetic profiles (Zhang et al. 2013).

The DNA fingerprints are stored in databases and sequences could be used for direct selection and identification of cotton hybrids and parents for future crop production programs. Moreover, the International Union for the Protection of new Varieties of Plants (UPOV) has encouraged the use of molecular markers in DUS testing for 
the identification of crop cultivars (UPOV-BMT 2002). Molecular markers are frequently used for effective selection, robust assessment of polymorphism and to explore the relativity of diverse genetic groups of cultivars with their wild relatives (Shah et al. 2009; Király et al. 2012). Previously, a restricted cotton gene pool has been classified by using Random amplified polymorphic DNA (RAPD) and amplified fragment length polymorphism (AFLP). SSR/Microsatellites are proven to be an ideal tool for DUS testing of new varieties because of high polymorphism, multi-allelic, co-dominant inheritance, good reproducibility, abundant distribution all over the genome and short amplification product and widely used for molecular characterization of genotypes to accelerate the effective selection (Jamil et al. 2020). About $>1000$ primers are identified from the cotton genome that is available in genome libraries (Nguyen et al. 2004; Yu et al. 2014).

Although some studies were conducted for DNA fingerprinting and genetic diversity assessment of cotton varieties in Pakistan previously (Mumtaz et al. 2010; Ullah et al. 2012). As far as Mumtaz et al. (2010) is concerned they have used RAPDs markers which are less reliable and non-reproducible. Ullah et al. (2012) although used SSR markers for DNA fingerprinting however genotypes used in their study were all primitive and number of SSR markers (104) used were relatively low which are unable to reveal genetic diversity in Pakistani cotton genotypes having narrow genetic base. Keeping in view of above said facts in our study we utilized 297 SSR markers for DNA fingerprinting and genetic diversity analysis of 25 cotton genotypes. Cluster analysis was conducted for estimation of genetic distance and to provide a reliable picture of a diverse grouping of genotypes for effective utilization of genetic information in cotton breeding programs. Structure analysis and dendrogram provides an insight into different sets of allelic richness in GM cotton genotypes. DNA fingerprints of GM cotton will provide a molecular basis to identify and authenticate the seed purity in the market.

\section{Materials and Methods}

\section{Plant material}

The research work was conducted at Agricultural Biotechnology Research Institute, Ayub Agricultural Research Institute Faisalabad, Pakistan for DNA fingerprinting of cotton genotypes for variety protection and registration under Plant Breeders Rights Rules 2017. Seeds of $25 \mathrm{GM}$ cotton genotypes (Pure-lines) were obtained from various institutes and stations from all over Punjab (Table 1) and were sown in pots in the greenhouse at $28^{\circ} \mathrm{C}$ following the standard agriculture practices. Each genotype was planted in 5 different pots wherein, each pot contained 2 seeds per genotype. After germination and seedling development till 3-4 leaves, 05 seedlings for each genotype were harvested and stored at $-40^{\circ} \mathrm{C}$ for DNA extraction.

\section{DNA isolation and PCR amplification}

DNA was isolated from $100 \mathrm{mg}$ of young leaves of GM cotton plants. The leaves were finely ground to powder using liquid nitrogen while DNA extraction was performed using the modified cetyltrimethylammonium bromide (CTAB) method (Allen et al. 2006). DNA samples for all genotypes were analyzed for the quality and quantity by NanoDrop spectrophotometer and also running them on Agarose Gel Electrophoresis as elaborated in our previous study (Jamil et al. 2020).

PCR was assembled using 2X DreamTaq Green PCR master mix ThermoFisher Scientific (K1082) as recommended by the manufacturer. The master mix aids us in direct loading the samples (PCR product) on gel and green dye does not cause any inconvenience during PCR reaction. For $2 \mathrm{X}$ we prepared $50 \mu \mathrm{L}$ PCR reaction mixture which was comprised of $25 \mu \mathrm{L}$ master mix, $200 \mathrm{ng}$ template DNA, $2 \mu M$ primer (forward \& reverse) and volume make up to $50 \mu \mathrm{L}$ using nuclease-free water. PCR profile was set as follows: 1 cycle of initial denaturation at $95^{\circ} \mathrm{C}$ for $5 \mathrm{~min}$, 35 cycles of denaturation at $95^{\circ} \mathrm{C}$ for $60 \mathrm{sec}$, annealing at $55^{\circ} \mathrm{C}$ for $1 \mathrm{~min}$ and extension at $72^{\circ} \mathrm{C}$ for $1 \mathrm{~min}$, the final extension for $5 \mathrm{~min}$ at $72^{\circ} \mathrm{C}$. The PCR product was stored at $4^{\circ} \mathrm{C}$ before electrophoresis.

\section{Poly acrylamide gel electrophoresis (PAGE)}

PCR amplicons were analyzed on Electrophoresis System model POWERPRO-3AMP (cleaver scientific limited) using 6\% (W/V) polyacrylamide gel and performed at 16 watts power. PAGE gel was stained by Silver nitrate staining for visualization according to the previously described staining protocol by (Caetano-Anolles 1997). Resulting patterns were analyzed and captured using Syngene trans-illuminator Gel Documentation System.

\section{Statistical analysis}

The size of the PCR amplicon for each genotype was estimated by comparing them with banding patterns of 50 bp DNA ladder (Invitrogen ${ }^{\mathrm{TM}}$ ) loaded in the PAGE gel and scored as 1 or 0 indicating the presence and absence of particular band size. NTSYSpc 2.0 software (Rohlf 1998) and Structure v. 2.3.4 were used for statistical analysis. Structure analysis utilizes a model-based Bayesian clustering approach to obtain pedigree information that provides different sets of allelic richness in cotton (Pritchard et al. 2000). NTSYSpc 2.0 uses an un-weighted pair Group Method of Arithmetic Means (UPGMA) to analyze multivariate diverse data and generates a dendrogram. The structure analyses were performed according to the following parameters: No admission model; K-value (110); Burn-in periods: 10,000; 2 number of in-iteration burns 10,000; Markov Chain Monte Carlo Simulation: 100,000. The structure was determined by using $\operatorname{LnP}(\mathrm{K})$ values against $\Delta \mathrm{K}$ values using Evanno Test (Evanno et al. 2005). 


\section{Results}

\section{SSR Polymorphism}

A total of 297 SSR markers evenly distributed on 26 cotton chromosomes were used for DNA fingerprinting and genetic diversity studies of 25 cotton genotypes (Table S1). Among 297 SSR markers, 25 markers were not amplified whereas 28 were monomorphic and the remaining 244 were polymorphic. The polymorphic 244 SSR markers amplified a total of 1537 alleles among which 1294 (84.18\%) were polymorphic and 243 alleles $(15.82 \%)$ were monomorphic. Minimum numbers of alleles 2 were amplified by 13 SSR markers namely BNL0347, BNL2570, BNL3103, BNL3140, CIR0208, CIR0210, DPL0058, DPL0156, DPL0163, DPL0273, JESPR85, MUCS0515 and TMB2920. Maximum numbers of alleles (19) were amplified by SSR marker BNL0137 among which 16 were polymorphic (Fig. 1). Maximum polymorphic alleles (PA) 18 were amplified by BNL-228. Lowest PIC value (0.08) was observed for DPL0156 whereas the highest PIC value (0.93) was recorded for seven SSR markers i.e., BNL0137, BNL0387, BNL3977, JESPR220, JESPR222, MGHES44 and TMB0471 collectively. The average number of alleles and polymorphic alleles was 6.3 and 5.3 respectively. The average PIC value was 0.73 whereas the size of amplicon ranged from 80 to $1000 \mathrm{bp}$ (Table 2).

\section{DNA fingerprinting}

Fifty-seven SSR markers were able to differentiate 25 cotton genotypes. There were two groups of genotypes concerning to DNA fingerprinting. Group, I comprised 19 genotypes that amplified unique alleles and were identifiable using single SSR marker. Group II was comprised of 06 genotypes which were not identifiable using unique alleles hence a two-step identification method was used for their identification (Table 3).

SSR marker BNL0119 amplified unique alleles for four genotypes i.e., MNH-1016, MNH-1020, BH-221, and IUB-13. Similarly, BNL0228 amplified unique allelic patterns for three genotypes i.e., MNH-886, FH-142, and IUB-13. IUB-13 was identifiable with the help of nine SSR markers, MNH-1016 was identifiable using six SSR markers, NIAB-878 amplified unique allelic pattern with five SSR markers, MNH-1020 and RH-668 with four markers, FH-326 and BH-221 with three markers, MNH886, VH-327, RH-647, RH-662, SLH-8, SLH-19 and BS-15 with two markers and VH-Gulzar, FH-142, SLH-06 and BH-201 by one marker as given in Table 3 .

\section{Genetic diversity studies}

The data of 244 polymorphic SSR markers were used to generate a UPGMA dendrogram to study the extent of genetic diversity among 25 cotton genotypes using the
SHAN similarity matrix. The similarity coefficient between 25 cotton genotypes varied from 0.63 to 0.91 . The dendrogram divided the genotypes into two distinct clusters (Fig. 2). The highest similarity was observed between FH152 and $\mathrm{FH}-142$ in cluster I sharing $91 \%$ of the genetic loci; whereas the lowest genetic similarity was observed between MNH-1020 and the rest of 24 genotypes sharing $63 \%$ of genetic loci in common. A domestic relationship exists between cultivar distribution and agro-ecological zones as is evident from the UPGMA dendrogram. Genotypes bred in different agro-ecological zones i.e., Multan, Sahiwal, Vehari, Faisalabad, and Bahawalpur tend to appear in the same clade in cluster analysis. However slight variation was observed for MNH-886, MNH-1020, RH-668, and BH-178 which did not follow geographical distribution (Fig. 2).

In most cases, cluster analysis results fitted well with pedigree parentage information. Genotypes lying in clade IA have a common parentage with one another except $\mathrm{VH}-$ 383 and VH-Gulzar. Similarly, varieties present in clade IB i.e., except SLH-08, SLH-19, and RH-668 have one parent in common with each other. Genotypes present in clade III i.e., $\mathrm{BH}-201$ and $\mathrm{BH}-221$ do not have common parentage; whereas genotypes present in cluster II i.e., IUB-13 and BS15 have shared parentage except for NIAB-878 and MNH886 (Fig. 2 and Table 1).

\section{Population structure studies}

The model-based Bayesian approach was used to infer the population structure of twenty-five genotypes of cotton. Population Structure was determined by plotting the LnP $(\mathrm{K})$ against $\Delta \mathrm{K}$ values through Evanno Test (Evanno et al. 2005). The highest $\Delta \mathrm{K}(10212.75)$ was observed for $\mathrm{K}=2$ suggesting the existence of two sub-populations i.e., population $1(\mathrm{P} 1)$ and population $2(\mathrm{P} 2)$. $\mathrm{P} 1$ was comprised of 21 genotypes whereas P2 was comprised of four genotypes. The expected heterozygosity was high among the individuals of P1 (0.132) as compared to P2 (0.10). Whereas genetic diversity among the individuals of P2 (Fst_2 = 0.82) was high as compared to P1 (Fst_1 = 0.69) (Fig. 3). The results suggested that all genotypes originated from the Government of Punjab Agriculture set up (Ayub Agricultural Research Institute, AARI) have similar blood except MNH-886. Similarly, varieties bred from Institute other than AARI i.e., IUB-13, BS-15, and NIAB-878 have similar blood.

\section{Discussion}

DNA fingerprinting and genetic diversity studies are of prime importance for germplasm maintenance, PBRs protection, and seed production in cotton (Santhy et al. 2019). For a cotton breeder, presence of genetic variability guides for interspecific or intraspecific hybridization (Sheidai et al. 2014). Estimation of genetic diversity and DNA fingerprinting characterizes the individuals and assign 
Table 1: List of genotypes used in the study along with pedigree/parentage information

\begin{tabular}{|c|c|c|}
\hline Institute Name & Variety name & Pedigree/Parentage \\
\hline \multirow[t]{4}{*}{ CRI, Multan } & MNH-1016 & MNH-786 (Non Bt.) $\times$ MNH-456 (Bt) \\
\hline & MNH-1020 & $96016 \times \mathrm{MNH}-456$ \\
\hline & MNH-1026 & C-26 (MNH-6070 $\times$ MNH-786) $\times$ FH-207 \\
\hline & MNH-886 & $($ FH-207 $\times$ MNH-770) $\times$ Bollgard-I \\
\hline \multirow[t]{3}{*}{ CRI, Khanpur } & RH-647 & RH-500 $\times$ FH-113 \\
\hline & RH-662 & $319 / 08 \times$ FH-113 \\
\hline & RH-668 & VH- $259 \times$ RH-620 \\
\hline \multirow[t]{3}{*}{ CRS, Sahiwal } & SLH-06 & SLH-334 × Neelum-121 \\
\hline & SLH-8 & SLS- $1 \times$ FH-142 \\
\hline & SLH-19 & SLH-336 × FH-114 \\
\hline \multirow[t]{4}{*}{ CRS, Vehari } & VH-327 & VH-289 × VH-291 (Bt.) \\
\hline & VH-Gulzar & VH-281 × VH-211 (Bt.) \\
\hline & VH-189 & VH-319 (Bt.) $\times$ FH-142 (Bt.) \\
\hline & VH-383 & $\mathrm{VH}-211(B t.) \times \mathrm{VH}-326$ \\
\hline \multirow[t]{3}{*}{ CRS, Bahawalpur } & BH-178 & $($ BH-162 $\times$ MNH-6070) $\times$ Neelum- 121 \\
\hline & BH-201 & (BH-172 × BH-126) × Neelum-121 \\
\hline & BH-221 & $(\mathrm{BH}-160 \times \mathrm{BH}-176) \times \mathrm{BH}-121$ \\
\hline \multirow[t]{5}{*}{ CRS, Faisalabad } & FH-142 & FH-114 × FH-207 \\
\hline & FH-Lalazar & FH-207 × NuCot-N-33B (Bollgard-I) \\
\hline & FH-152 & FH-207 × FH-113 \\
\hline & FH-326 & FH-942 $\times$ FH-114 \\
\hline & FH-490 & FH-113 × FH-2006 \\
\hline Islamia University Bahawalpur & IUB-13 & IUB-09 $\times$ MNH-789 \\
\hline Bandesha Seed Corporation & BS-15 & IB $2009 \times$ MNH-786 \\
\hline NIAB, Faisalabad & NIAB-878 & B-111 × NIAB-Kiran \\
\hline
\end{tabular}

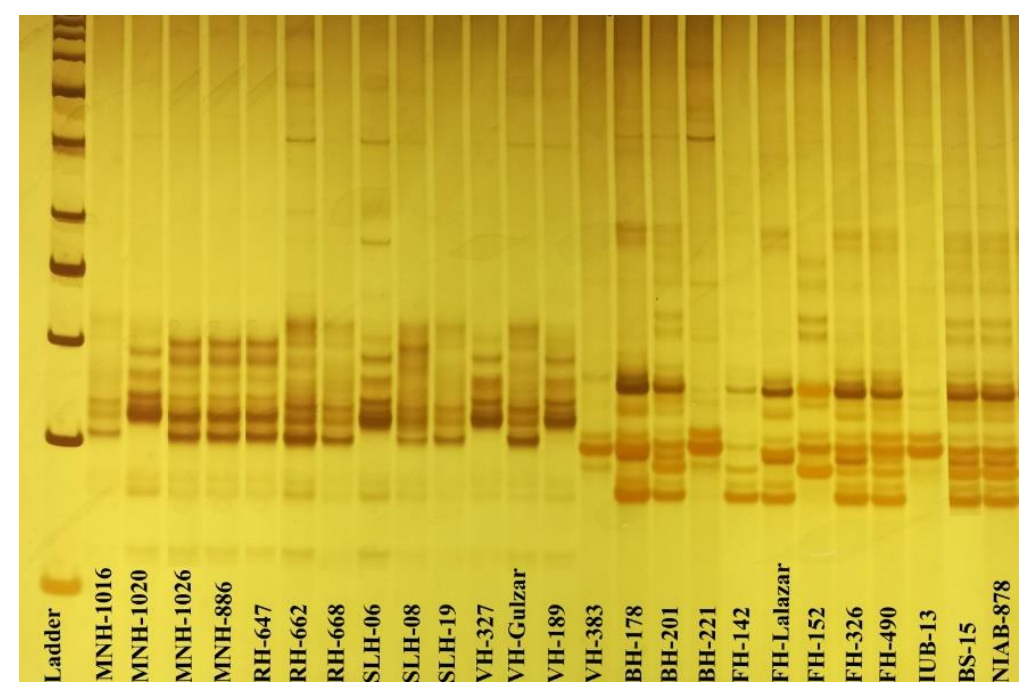

Fig. 1: The amplification product of BNL-0137. The most informative SSR marker with 19 number of alleles among which 16 are polymorphic

them to different heterotic groups for the choice of parental genotypes for hybridization-based breeding programs (Noormohammadi et al. 2018; Ul-Allah et al. 2019).

In past different types of molecular markers i.e., RFLPs, RAPDs, AFLPs, ISSRs, and SSR were used for DNA fingerprinting and genetic diversity studies in cotton (Becelaere et al. 2005; Khan et al. 2010; Badigannavar et al. 2012; Noormohammadi et al. 2013). However, the present study evidenced that SSR markers are still an effective tool to differentiate cotton cultivars due to high polymorphism, ease of use, and high reproducibility. However, to exploit genetic variation we have to use a very large set of SSR markers which is an indication of a narrow genetic base in the cotton germplasm (Fig. 2).

Unlike most of the previous studies (Zhang et al. 2013; Noormohammadi et al. 2018), not all the cotton varieties produced unique allelic patterns as six varieties in the present study did not amplify unique bands. Some informative SSR markers showing a high level of polymorphism are BNL0137, BNL-228, BNL0387 TMB0471, JEPSR220 BNL0140, CIR0251, BNL2616, JESPR222, BNL3590, and BNL3977. 
Table 2: List of SSR markers used along with Polymorphism information, Number of Alleles (NOA) Polymorphic Alleles (PA), Polymorphic Information Contents (PIC) and annealing temperature (TA)

\begin{tabular}{|c|c|c|c|c|c|c|c|c|c|c|c|}
\hline Sr. No. & Name & Polymorphism & $\mathrm{NOA}$ & PA & PIC & Sr. No. & Name & Polymorphism & $\mathrm{NOA}$ & PA & PIC \\
\hline 1. & BNL0113 & Polymorphic & 5 & 5 & 0.78 & 150. & CIR0099 & Monomorphic & & & \\
\hline 2. & BNL0116 & Polymorphic & 4 & 4 & 0.66 & 151. & CIR0133 & Polymorphic & 8 & 6 & 0.86 \\
\hline 3. & BNL0117 & Polymorphic & 8 & 7 & 0.87 & 152. & CIR0135 & Not Amplified & & & \\
\hline 4. & BNL0118 & Polymorphic & 3 & 3 & 0.61 & 153. & CIR0143 & Polymorphic & 5 & 5 & 0.80 \\
\hline 5. & BNL0119 & Polymorphic & 14 & 13 & 0.89 & 154. & CIR0169 & Monomorphic & & & \\
\hline 6. & BNL0128 & Polymorphic & 5 & 5 & 0.78 & 155. & CIR0180 & Monomorphic & & & \\
\hline 7. & BNL0134 & Monomorphic & & & & 156. & CIR0181 & Monomorphic & & & \\
\hline 8. & BNL0135 & Polymorphic & 4 & 2 & 0.75 & 157. & CIR0203 & Polymorphic & 7 & 5 & 0.70 \\
\hline 9. & BNL0137 & Polymorphic & 19 & 16 & 0.93 & 158. & CIR0208 & Polymorphic & 2 & 2 & 0.50 \\
\hline 11. & BNL0148 & Polymorphic & 6 & 6 & 0.83 & 160. & CIR0221 & Polymorphic & 3 & 1 & 0.67 \\
\hline 12. & BNL0150 & Polymorphic & 10 & 8 & 0.90 & 161. & CIR0224 & Polymorphic & 4 & 3 & 0.75 \\
\hline 13. & BNL0153 & Polymorphic & 7 & 7 & 0.85 & 162. & CIR0246 & Polymorphic & 6 & 6 & 0.71 \\
\hline 14. & BNL0162A & Not Amplified & & & & 163. & CIR0251 & Polymorphic & 14 & 14 & 0.92 \\
\hline 15. & BNL0174 & Polymorphic & 10 & 6 & 0.89 & 164. & CIR0272 & Polymorphic & 4 & 4 & 0.75 \\
\hline 16. & BNL0193 & Polymorphic & 5 & 5 & 0.80 & 165. & CIR0294 & Polymorphic & 4 & 4 & 0.75 \\
\hline 17. & BNL0197 & Polymorphic & 3 & 3 & 0.67 & 166. & CIR0307 & Polymorphic & 10 & 10 & 0.89 \\
\hline 18. & BNL0206 & Polymorphic & 7 & 5 & 0.86 & 167. & CIR036 & Polymorphic & 8 & 5 & 0.87 \\
\hline 19. & BNL0218 & Polymorphic & 10 & 10 & 0.89 & 168. & CIR0372 & Polymorphic & 7 & 7 & 0.85 \\
\hline 21. & BNL0220 & Polymorphic & 3 & 3 & 0.53 & 170. & CIR0413 & Polymorphic & 3 & 3 & 0.67 \\
\hline 22. & BNL0223 & Polymorphic & 10 & 9 & 0.88 & 171. & CIR0415 & Polymorphic & 5 & 3 & 0.78 \\
\hline 23. & BNL0225 & Polymorphic & 12 & 10 & 0.91 & 172. & CIR049 & Polymorphic & 3 & 3 & 0.67 \\
\hline 24. & BNL0226 & Not Amplified & & & & 173. & CIR060 & Polymorphic & 4 & 1 & 0.75 \\
\hline 25. & BNL0228 & Polymorphic & 18 & 18 & 0.92 & 174. & CIR062 & Polymorphic & 4 & 3 & 0.75 \\
\hline 26. & BNL0234 & Polymorphic & 7 & 7 & 0.85 & 175. & CIR122 & Polymorphic & 7 & 5 & 0.86 \\
\hline 27. & BNL0236 & Polymorphic & 4 & 4 & 0.75 & 176. & CM14 & Not Amplified & & & \\
\hline 28. & BNL0237 & Polymorphic & 4 & 3 & 0.72 & 177. & CM17 & Not Amplified & & & \\
\hline 29. & BNL0244 & Polymorphic & 6 & 5 & 0.82 & 178. & CM32 & Not Amplified & & & \\
\hline 30. & BNL0285 & Polymorphic & 7 & 6 & 0.86 & 179. & CM4 & Polymorphic & 6 & 5 & 0.83 \\
\hline 31. & BNL0300 & Polymorphic & 4 & 4 & 0.75 & 180. & CM45 & Polymorphic & 13 & 4 & 0.92 \\
\hline 32. & BNL0329 & Polymorphic & 9 & 8 & 0.85 & 181. & CM6 & Not Amplified & & & \\
\hline 33. & BNL0341 & Polymorphic & 9 & 9 & 0.88 & 182. & CM60 & Polymorphic & 7 & 3 & 0.86 \\
\hline 34. & BNL0343 & Polymorphic & 4 & 4 & 0.74 & 183. & CM63 & Not Amplified & & & \\
\hline 35. & BNL0347 & Polymorphic & 2 & 2 & 0.50 & 184. & CM66 & Monomorphic & & & \\
\hline 36. & BNL0358 & Polymorphic & 5 & 1 & 0.80 & 185. & CM67 & Polymorphic & 7 & 7 & 0.76 \\
\hline 37. & BNL0379 & Not Amplified & & & & 186. & CM68 & Not Amplified & & & \\
\hline 38. & BNL0386 & Polymorphic & 10 & 10 & 0.88 & 187. & $\mathrm{CM} 7$ & Not Amplified & & & \\
\hline 42. & BNL0448 & Polymorphic & 9 & 3 & 0.87 & 191. & DPL0058 & Polymorphic & 2 & 1 & 0.50 \\
\hline 43. & BNL0530 & Not Amplified & & & & 192. & DPL0079 & Polymorphic & 4 & 3 & 0.67 \\
\hline 44. & BNL0584 & Polymorphic & 3 & 3 & 0.66 & 193. & DPL0133 & Polymorphic & 5 & 4 & 0.79 \\
\hline 45. & BNL0597 & Polymorphic & 4 & 1 & 0.75 & 194. & DPL0149 & Polymorphic & 5 & 5 & 0.70 \\
\hline 46. & BNL0686 & Monomorphic & & & & 195. & DPL0156 & Polymorphic & 2 & 2 & 0.08 \\
\hline 47. & BNL0827 & Polymorphic & 3 & 2 & 0.67 & 196. & DPL0163 & Polymorphic & 2 & 2 & 0.50 \\
\hline 48. & BNL0829 & Polymorphic & 6 & 6 & 0.83 & 197. & DPL0264 & Polymorphic & 6 & 4 & 0.83 \\
\hline 49. & BNL0830 & Polymorphic & 2 & 2 & 0.27 & 198. & DPL0273 & Polymorphic & 2 & 2 & 0.50 \\
\hline 50. & BNL0834 & Polymorphic & 8 & 8 & 0.88 & 199. & DPL0348 & Monomorphic & & & \\
\hline 51. & BNL0891 & Polymorphic & 5 & 5 & 0.80 & 200. & DPL0385 & Polymorphic & 4 & 4 & 0.75 \\
\hline 52. & BNL0946 & Polymorphic & 5 & 5 & 0.76 & 201. & DPL0443 & Monomorphic & & & \\
\hline 53. & BNL1017 & Monomorphic & & & & 202. & DPL0489 & Monomorphic & & & \\
\hline 54. & BNL1161 & Polymorphic & 8 & 4 & 0.87 & 203. & DPL0528 & Polymorphic & 4 & 3 & 0.75 \\
\hline 55. & BNL1253 & Polymorphic & 5 & 5 & 0.77 & 204. & DPL0534 & Polymorphic & 4 & 3 & 0.75 \\
\hline 56. & BNL1317 & Polymorphic & 8 & 8 & 0.84 & 205. & DPL0542 & Polymorphic & 7 & 4 & 0.82 \\
\hline 57. & BNL1403 & Polymorphic & 3 & 3 & 0.67 & 206. & HAU0119 & Polymorphic & 7 & 3 & 0.82 \\
\hline 58. & BNL1417 & Polymorphic & 7 & 7 & 0.84 & 207. & JESPR0102 & Monomorphic & & & \\
\hline 59. & BNL1418 & Monomorphic & 4 & 0 & 0.75 & 208. & JESPR0135 & Polymorphic & 9 & 9 & 0.89 \\
\hline 60. & BNL1441 & Polymorphic & 5 & 5 & 0.80 & 209. & JESPR0232 & Polymorphic & 9 & 7 & 0.89 \\
\hline 61. & BNL1531 & Polymorphic & 6 & 3 & 0.83 & 210. & JESPR0240 & Monomorphic & & & \\
\hline 62. & BNL1592 & Polymorphic & 2 & 2 & 0.50 & 211. & JESPR1 & Polymorphic & 4 & 3 & 0.70 \\
\hline 63. & BNL1597 & Polymorphic & 9 & 7 & 0.88 & 212. & JESPR100 & Polymorphic & 4 & 4 & 0.72 \\
\hline 64. & BNL1605 & Not Amplified & & & & 213. & JESPR101 & Not Amplified & & & \\
\hline 65. & BNL1667 & Polymorphic & 5 & 4 & 0.79 & 214. & JESPR103 & Polymorphic & 8 & 8 & 0.87 \\
\hline 66. & BNL1681 & Not Amplified & & & & 215. & JESPR108 & Polymorphic & 3 & 3 & 0.63 \\
\hline 67. & BNL1688 & Polymorphic & 6 & 1 & 0.83 & 216. & JESPR114 & Polymorphic & 11 & 11 & 0.89 \\
\hline 68. & BNL1694 & Polymorphic & 5 & 3 & 0.80 & 217. & JESPR134 & Polymorphic & 10 & 9 & 0.88 \\
\hline 69. & BNL2443 & Monomorphic & & & & 218. & JESPR 153 & Polymorphic & 11 & 11 & 0.88 \\
\hline 70. & BNL2448 & Polymorphic & 6 & 5 & 0.83 & 219. & JESPR156 & Polymorphic & 4 & 4 & 0.71 \\
\hline 71. & BNL2527 & Polymorphic & 11 & 11 & 0.91 & 220. & JESPR160 & Polymorphic & 3 & 2 & 0.64 \\
\hline 72. & BNL2544 & Polymorphic & 5 & 5 & 0.80 & 221. & JESPR173 & Polymorphic & 6 & 6 & 0.83 \\
\hline 73. & BNL2564 & Polymorphic & 4 & 4 & 0.75 & 222. & JESPR178 & Polymorphic & 5 & 5 & 0.80 \\
\hline 74. & BNL2570 & Polymorphic & 2 & 2 & 0.50 & 223. & JESPR185 & Polymorphic & 5 & 5 & 0.79 \\
\hline 75. & BNL2572 & Polymorphic & 4 & 4 & 0.75 & 224. & JESPR186 & Not Amplified & & & \\
\hline
\end{tabular}


DNA Fingerprinting of Cotton / Intl J Agric Biol, Vol 25, No 4, 2021

Table 2: Continue

\begin{tabular}{|c|c|c|c|c|c|c|c|c|c|c|c|}
\hline 76. & BNL2590 & Polymorphic & 9 & 6 & 0.88 & 225 . & JESPR194 & Polymorphic & 8 & 8 & 0.87 \\
\hline 77. & BNL2597 & Polymorphic & 5 & 3 & 0.80 & 226. & JESPR200 & NA & & & \\
\hline 78. & BNL2599 & Polymorphic & 3 & 3 & 0.67 & 227. & JESPR202 & NA & & & \\
\hline 79. & BNL2616 & Polymorphic & 15 & 14 & 0.91 & 228. & JESPR205 & Polymorphic & 6 & 4 & 0.82 \\
\hline 80. & BNL2632 & Polymorphic & 12 & 12 & 0.88 & 229. & JESPR209 & Polymorphic & 2 & 2 & 0.50 \\
\hline 81. & BNL2634 & Polymorphic & 12 & 10 & 0.91 & 230. & JESPR215 & Polymorphic & 13 & 13 & 0.91 \\
\hline 82. & BNL2652 & Polymorphic & 4 & 4 & 0.73 & 231. & JESPR218 & Monomorphic & & & \\
\hline 83. & BNL2681 & Polymorphic & 4 & 2 & 0.75 & 232. & JESPR220 & Polymorphic & 15 & 15 & 0.93 \\
\hline 84. & BNL2700 & Polymorphic & 10 & 9 & 0.89 & 233. & JESPR222 & Polymorphic & 14 & 14 & 0.93 \\
\hline 85. & BNL2750 & Polymorphic & 2 & 1 & 0.50 & 234. & JESPR227 & Polymorphic & 6 & 6 & 0.79 \\
\hline 86. & BNL2762 & Polymorphic & 6 & 6 & 0.83 & 235 . & JESPR229 & Monomorphic & & & \\
\hline 87. & BNL2772 & Polymorphic & 5 & 4 & 0.80 & 236. & JESPR232 & Polymorphic & 7 & 4 & 0.83 \\
\hline 88. & BNL2827 & Monomorphic & & & & 237. & JESPR236 & Polymorphic & 7 & 4 & 0.83 \\
\hline 89. & BNL2835 & Polymorphic & 11 & 6 & 0.91 & 238 & JESPR242 & Polymorphic & 6 & 6 & 0.82 \\
\hline 90. & BNL2882 & Polymorphic & 9 & 5 & 0.89 & 239. & JESPR244 & Monomorphic & & & \\
\hline 91. & BNL2986 & Monomorphic & & & & 240 & JESPR246 & Polymorphic & 11 & 11 & 0.90 \\
\hline 92. & BNL3029 & Polymorphic & 3 & 3 & 0.67 & 241. & JESPR250 & Polymorphic & 8 & 8 & 0.78 \\
\hline 93. & BNL3034 & Polymorphic & 4 & 2 & 0.74 & 242 & JESPR270 & Polymorphic & 7 & 3 & 0.86 \\
\hline 94. & BNL3071 & Not Amplified & & & & 243. & JESPR272 & Not Amplified & & & \\
\hline 95. & BNL3090 & Polymorphic & 6 & 3 & 0.83 & 244. & JESPR291 & Monomorphic & & & \\
\hline 96. & BNL3103 & Polymorphic & 2 & 2 & 0.50 & 245 . & JESPR292 & Polymorphic & 3 & 2 & 0.49 \\
\hline 97. & BNL3140 & Polymorphic & 2 & 2 & 0.50 & 246. & JESPR296 & Polymorphic & 4 & 4 & 0.74 \\
\hline 98. & BNL3147 & Polymorphic & 4 & 4 & 0.75 & 247. & JESPR 310 & Polymorphic & 6 & 5 & 0.83 \\
\hline 99. & BNL3255 & Polymorphic & 9 & 4 & 0.88 & 248. & JESPR42 & Polymorphic & 11 & 7 & 0.90 \\
\hline 100. & BNL3279 & Polymorphic & 7 & 7 & 0.85 & 249. & JESPR80 & Not Amplified & & & \\
\hline 101. & BNL3319 & Polymorphic & 5 & 5 & 0.78 & 250 & JESPR84 & Polymorphic & 8 & 5 & 0.87 \\
\hline 102. & BNL3324 & Polymorphic & 3 & 3 & 0.67 & 251. & JESPR85 & Polymorphic & 2 & 2 & 0.50 \\
\hline 103. & BNL3345 & Polymorphic & 5 & 4 & 0.63 & 252. & JESPR94 & Polymorphic & 3 & 3 & 0.65 \\
\hline 104. & BNL3379 & Polymorphic & 7 & 3 & 0.86 & 253. & JESPR95 & Polymorphic & 7 & 7 & 0.84 \\
\hline 105. & BNL3383 & Polymorphic & 7 & 7 & 0.85 & 254. & MGHES11a & Polymorphic & 6 & 6 & 0.83 \\
\hline 106. & BNL3408 & Polymorphic & 10 & 10 & 0.89 & 255. & MGHES11b & Polymorphic & 4 & 4 & 0.73 \\
\hline 107. & BNL3414 & Polymorphic & 5 & 3 & 0.80 & 256. & MGHES18 & Polymorphic & 3 & 3 & 0.65 \\
\hline 108. & BNL3432 & Polymorphic & 5 & 5 & 0.80 & 257. & MGHES24 & Polymorphic & 11 & 11 & 0.91 \\
\hline 109. & BNL3449 & Polymorphic & 8 & 8 & 0.86 & 258. & MGHES30a & Monomorphic & & & \\
\hline 110. & BNL3452 & Polymorphic & 5 & 5 & 0.80 & 259. & MGHES32 & Not Amplified & & & \\
\hline 111. & BNL3523 & Polymorphic & 7 & 2 & 0.85 & 260. & MGHES40 & Polymorphic & 7 & 7 & 0.85 \\
\hline 112. & BNL3556 & Monomorphic & & & & 261. & MGHES41 & Polymorphic & 9 & 8 & 0.88 \\
\hline 113. & BNL3558 & Polymorphic & 3 & 3 & 0.67 & 262. & MGHES44 & Polymorphic & 14 & 13 & 0.93 \\
\hline 114. & BNL3563 & Polymorphic & 7 & 3 & 0.86 & 263. & MGHES46 & Not Amplified & & & \\
\hline 115. & BNL3582 & Polymorphic & 3 & 2 & 0.66 & 264. & MGHES48 & Polymorphic & 13 & 13 & 0.92 \\
\hline 116. & BNL3590 & Polymorphic & 14 & 14 & 0.92 & 265. & MGHES59 & Polymorphic & 3 & 3 & 0.64 \\
\hline 117. & BNL3592 & Polymorphic & 5 & 3 & 0.80 & 266. & MGHES6 & Polymorphic & 3 & 3 & 0.56 \\
\hline 118. & BNL3599 & Polymorphic & 13 & 13 & 0.92 & 267. & MGHES70 & Polymorphic & 8 & 6 & 0.80 \\
\hline 119. & BNL3601 & Polymorphic & 4 & 2 & 0.71 & 268. & MGHES71 & Polymorphic & 5 & 5 & 0.79 \\
\hline 120. & BNL3646 & Polymorphic & 3 & 1 & 0.67 & 269. & MGHES73 & Polymorphic & 11 & 11 & 0.89 \\
\hline 121. & BNL3649 & Monomorphic & & & & 270 & MGHES75 & Polymorphic & 5 & 5 & 0.76 \\
\hline 122. & BNL3661 & Polymorphic & 8 & 5 & 0.86 & 271. & MGHES76 & Polymorphic & 6 & 6 & 0.82 \\
\hline 123. & BNL3799 & Monomorphic & & & & 272. & MUCS0515 & Polymorphic & 2 & 1 & 0.50 \\
\hline 124. & BNL3860 & Polymorphic & 13 & 8 & 0.92 & 273. & MUSB1121 & Polymorphic & 5 & 5 & 0.71 \\
\hline 125. & BNL3903 & Polymorphic & 4 & 1 & 0.75 & 274. & NAU0808 & Polymorphic & 5 & 3 & 0.78 \\
\hline 126. & BNL3935 & Polymorphic & 11 & 7 & 0.91 & 275 & NAU2083 & Polymorphic & 9 & 9 & 0.83 \\
\hline 127. & BNL3948 & Polymorphic & 3 & 1 & 0.64 & 276. & NAU2540 & Polymorphic & 5 & 5 & 0.73 \\
\hline 128. & BNL3976 & Polymorphic & 3 & 1 & 0.67 & 277. & NAU2580 & Polymorphic & 4 & 1 & 0.75 \\
\hline 129. & BNL3977 & Polymorphic & 14 & 14 & 0.93 & 278. & NAU2679 & Polymorphic & 4 & 4 & 0.62 \\
\hline 130. & BNL3985 & Polymorphic & 4 & 4 & 0.74 & 279. & NAU2715 & Polymorphic & 4 & 1 & 0.73 \\
\hline 131. & BNL3988 & Polymorphic & 6 & 6 & 0.83 & 280. & NAU2954 & Polymorphic & 5 & 5 & 0.80 \\
\hline 132. & BNL3995 & Polymorphic & 3 & 3 & 0.67 & 281. & NAU3100 & Polymorphic & 8 & 8 & 0.87 \\
\hline 133. & BNL4011 & Polymorphic & 3 & 2 & 0.62 & 282. & NAU6672 & Polymorphic & 4 & 3 & 0.75 \\
\hline 134. & BNL4015 & Not Amplified & & & & 283. & TMB0034 & Not Amplified & & & \\
\hline 135. & BNL4030 & Not Amplified & & & & 284. & TMB0471 & Polymorphic & 16 & 15 & 0.93 \\
\hline 136. & BNL4078 & Polymorphic & 3 & 3 & 0.67 & 285. & TMB0603 & Polymorphic & 4 & 1 & 0.75 \\
\hline 137. & BNL4080 & Monomorphic & & & & 286. & TMB0770 & Polymorphic & 5 & 5 & 0.80 \\
\hline 138. & BNL4082 & Polymorphic & 6 & 6 & 0.83 & 287. & TMB1296 & Polymorphic & 6 & 4 & 0.83 \\
\hline 139. & BNL4092 & Polymorphic & 7 & 7 & 0.86 & 288. & TMB1356 & Polymorphic & 6 & 1 & 0.83 \\
\hline 140. & BNL786 & Polymorphic & 7 & 7 & 0.78 & 289. & TMB1456 & Monomorphic & & & \\
\hline 141. & BNL834 & Polymorphic & 7 & 7 & 0.85 & 290. & TMB1548 & Polymorphic & 6 & 6 & 0.83 \\
\hline 142. & CGR5641 & Polymorphic & 4 & 2 & 0.74 & 291. & TMB1638 & Polymorphic & 4 & 3 & 0.75 \\
\hline 143. & CGR6692 & Polymorphic & 4 & 4 & 0.75 & 292. & TMB1639 & Polymorphic & 6 & 2 & 0.82 \\
\hline 144. & CGR6692 & Polymorphic & 3 & 2 & 0.63 & 293. & TMB1838 & Polymorphic & 3 & 1 & 0.66 \\
\hline 145. & CGR6824 & Polymorphic & 7 & 7 & 0.80 & 294. & TMB1919 & Polymorphic & 5 & 5 & 0.80 \\
\hline 146. & CIR0054 & Polymorphic & 4 & 4 & 0.75 & 295. & TMB2920 & Polymorphic & 2 & 2 & 0.50 \\
\hline 147. & CIR0061 & Polymorphic & 7 & 7 & 0.85 & 296. & TMB2945 & Polymorphic & 3 & 2 & 0.66 \\
\hline 148. & CIR0082 & Polymorphic & 11 & 11 & 0.91 & 297. & TMH05 & Monomorphic & & & \\
\hline 149. & CIR0094 & Polymorphic & 10 & 9 & 0.85 & & & & & & \\
\hline
\end{tabular}

Note: Annealing temperature of all primers was $55^{\circ} \mathrm{C}$ 
Table 3: List of SSR markers that can distinguish twenty-five varieties of cotton using direct or indirect method

\begin{tabular}{ll}
\hline Genotypes & DNA Fingerprints \\
\hline MNH-886 & BNL0228, MGHES24 \\
MNH-1016 & BNL0123, CIR0203, NAU2679, BNL0119, MGHES75, JESPR153 \\
MNH-1020 & BNL0119, BNL0391, BNL2634, JESPR232 \\
MNH-1026 & Identifiable using pair of SSR markers (BNL2632 \& BNL0123) and (BNL0341 \& CIR0230) \\
VH-327 & MGHES75, JESPR215 \\
VH-Gulzar & BNL0134 \\
VH-189 & Identifiable using pair of SSR markers (BNL0830 \& BNL0119) and (DPL0153 \& BNL0134) \\
VH-383 & Identifiable using pair of SSR markers (BNL3601 \& BNL0119) and (BNL3449 \& CIR0391) \\
FH-142 & BNL0228 \\
FH-Lalazar & Identifiable using pair of SSR markers (BNL0830 \& JESPR232) and (BNL0237 \& CIR0203) \\
FH-152 & Identifiable using pair of SSR markers (BNL834 \& BNL1253) and ( BNL786 \& BNL448) \\
FH-326 & DPL0542, CIR0246, DPL0149 \\
FH-490 & Identifiable using pair of SSR markers ( TMB2926 \& BNL0123) and ( BNL3988 \& JESPR232) \\
RH-647 & BNL1253, DPL0133 \\
RH-662 & BNL2616, MGHES73 \\
RH-668 & DPL0156, CIR0094, UAU0119, BNL0329 \\
SLH-06 & BNL0448 \\
SLH-8 & MGHES6, JESPR153 \\
SLH-19 & BNL0137, JESPR250 \\
BH-178 & Identifiable using pair of SSR markers ( BNL1592 \& BNL3529) and (BNL0329 \& JESPR153) \\
BH-201 & JESPR236 \\
BH-221 & BNL3529, BNL0220, BNL0119 \\
NIAB-878 & NAU2083, BNL2540, BNL2599, BNL0140, JESPR114 \\
IUB-13 & HAU0119, CIR0307, BNL4082, BNL0390, BNL0150, BNL0228, BNL0119, BNL0236, JESPR100 \\
BS-15 & BNL2835, MGHES24
\end{tabular}

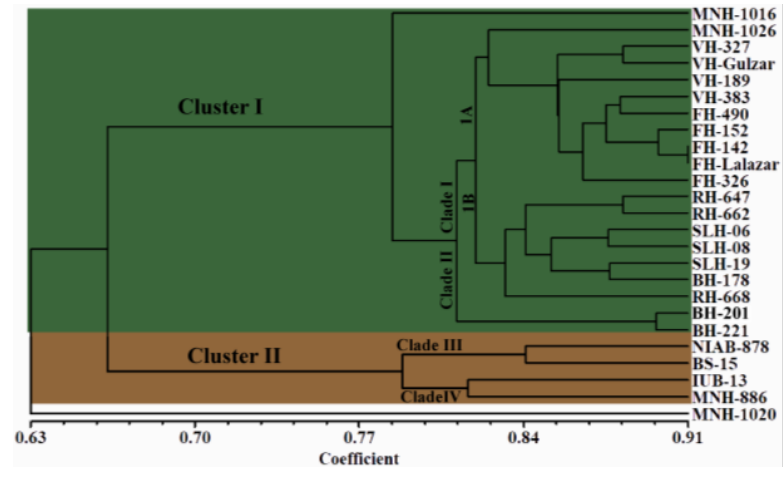

Fig. 2: Dendrogram of 25 cotton genotypes generated using data of 244 polymorphic SSR markers through SHAN similarity matrix and unweighted pair group method

These SSR markers may be used for DNA fingerprinting and genetic diversity studies in the future. Our results are in line with the previous studies (Bertini et al. 2006; Lacape et al. 2007) which also reported informative SSR markers for genotyping and genetic diversity studies.

The average alleles and polymorphic alleles per locus 6.3 and 5.3 respectively reported in our study were higher than many of previously published studies. Zhu et al. (2019) reported 6.02 alleles per locus in a study comprising of 557 G. hirsutism accessions. Javaid et al. (2017) reported 3.72 alleles per locus in a study of genetic diversity in 22 cotton accessions using 30 SSR markers. Similarly, Gurmessa (2019) reported 3.8 alleles per locus with 0.50 PIC value. Whereas according to our knowledge only one study of McCarty et al. (2018) reported a high number of alleles (7.9) per locus. This is expected because they used landraces and genetic diversity in landraces is more than the cultivated varieties. However, Average PIC value reported in our study
0.73 is highest among all the previously published reports. High number of alleles in our study and high PIC value corresponds to large set of SSR markers used in our study (Table 2).

Different studies have reported a continuous decline in cotton productivity in Pakistan for the past 03 years (Ashraf et al. 2018; Ali et al. 2019b; Rana et al. 2020; Jamil et al. 2021). Whereas some model-based future predictions are pointing out that this trend will continue for another four to five years (Ashraf et al. 2018). The question arises what are major factors that are hampering cotton productivity? One possible answer to this question is the lack of genetic divergence in the cultivated cotton genotypes as proved through our results. The varieties used in this study covered almost $60 \%$ of the cropped area under cotton cultivation. However, when it comes to genetics there are only two types of blood as is evident from structure analysis. About $84 \%$ of genotypes (21) have similar genetic makeup and formulate P1 (Fig. 3). The pedigree parentage dictates that five genotypes have FH-207 as a common parent. The same is the case with Neelum-121 which is used as a parent in breeding of three genotypes and many other such examples exists in Table 1.

The pressure for higher productivity in cotton farming and continuous artificial selection have narrows down the genetic base which is a major hurdle for successful cotton breeding programs (Noormohammadi et al. 2018). It happens when you start with a broad genetic base but if the base material (Pedigree/Parentage) is itself has narrow genetic makeup as is our case, what will be its outcome? Crops will be more prone to biotic and abiotic stresses as is happening in cotton i.e., Whitefly (Ahmad and Akhtar 2018), Jassids, aphids, thrips (Akhtar et al. 2018) and bollworms (Ahmad et al. 2019) heavily infest almost all 

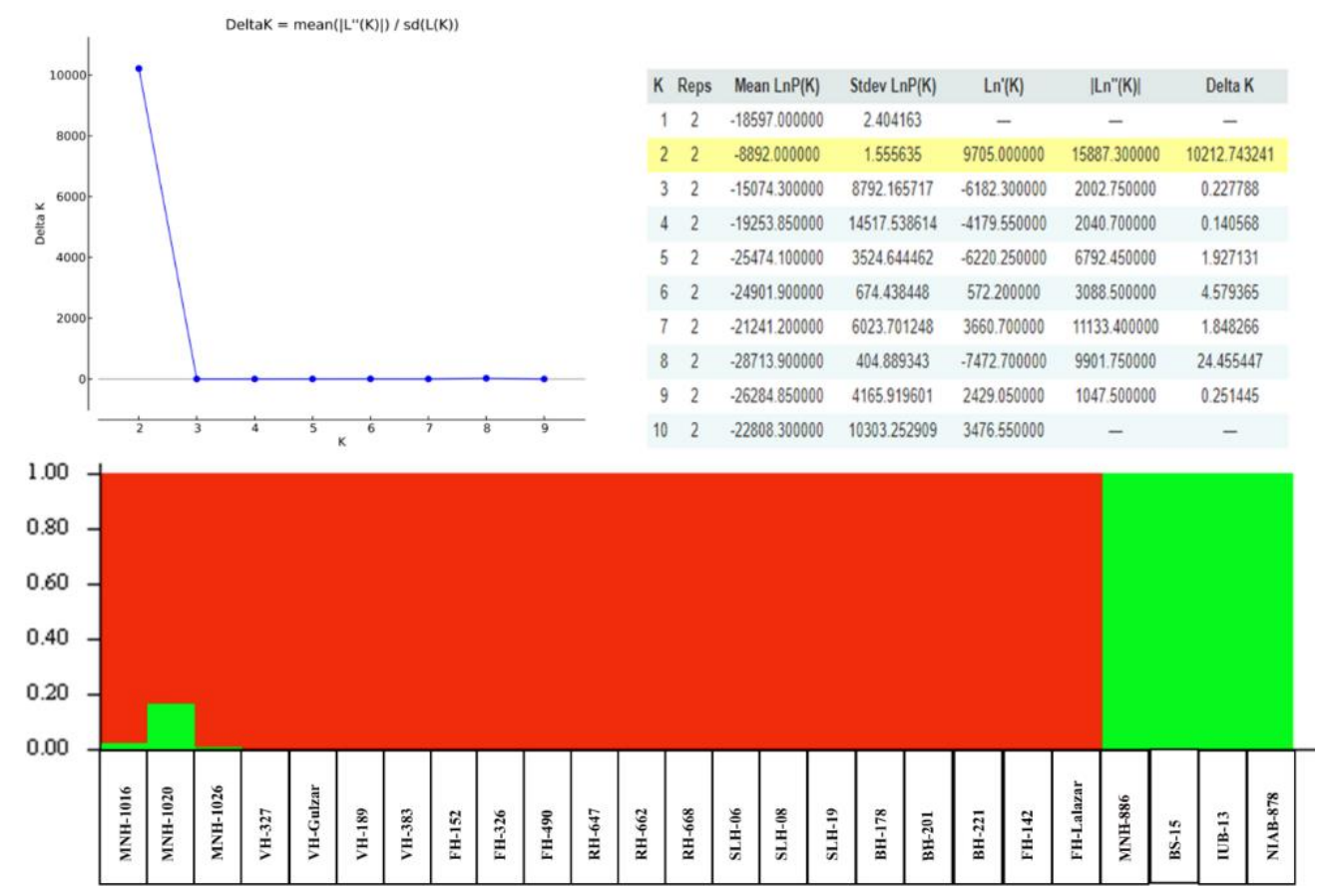

Fig. 3: Structure Analysis of Cotton varieties grown in Punjab Pakistan. Parameters: no admission model; K = 02; 10,000 Burn-in period; 100000 Rep

cotton varieties and cause almost $15-20 \%$ crop losses every year (Khan et al. 2016; Khanzada et al. 2016). Our breeding and selection efforts have narrowed down genetic base which needs to be broadened for the revival of cotton (Khanzada et al. 2016; Ali et al. 2019a).

\section{Conclusion}

DNA fingerprints were developed for twenty-five GM cotton genotypes grown in Punjab. The genetic diversity studies grouped the genotypes to two distinct groups P1 (20 genotypes), P2 (04 genotypes) whereas MNH-1020 did not follow clustering. The genetic makeup of cotton genotypes used in the study was narrow. We reported polymorphism information of 244 polymorphic SSR markers and proposed a core set of markers for future DNA fingerprinting and genetic diversity studies. Our study will provide a platform for the protection of Plant Breeders Rights and will help in registration of variety under Plant Breeders Rights Registry.

\section{Acknowledgments}

The Authors are highly thankful to Punjab Agricultural Research Board for providing funding to conduct this research work through PARB Project No. 908 entitled "DNA barcoding/fingerprinting for identification of Cotton, Wheat, Maize, Potato, Tomato and Date Palm varieties". Mr. Baber Ali Lab Assistant, for technical assistance during research work. The Cotton Research Stations and Institute across the Province for providing the plant material and technical support.

\section{Author Contributions}

SJ, RS, MZI and SUR obtained funding, SJ, RS and EY conducted research experimentation, SJ, RS conducted statistical data analysis, SJ, RS and EY drafted the manuscript, SUR and MZI critically reviewed the manuscript. SJ, RS, MZI and SUR supervised the research experimentation and all process. SJ and RS corresponded to journal for submission and review process.

\section{Conflict of Interest}

The authors declare no conflict of interest among them

\section{Data Availability declaration}

We hereby declare that data, primary or supplementary related to this article, are available with the corresponding author and will be produced on demand

\section{References}

Ahmad M, KP Akhtar (2018). Susceptibility of cotton whitefly Bemisia tabaci (Hemiptera: Aleyrodidae) to diverse pesticides in Pakistan. $J$. Econ Entomol 111:1834-1841

Ahmad M, B Rasool, M Ahmad, DA Russell (2019). Resistance and Synergism of Novel Insecticides in Field Populations of Cotton Bollworm Helicoverpa armigera (Lepidoptera: Noctuidae) in Pakistan. J Econ Entomol 112:859-871

Akhtar ZR, U Irshad, M Majid, Z Saeed, H Khan, AA Anjum, A Noreen, M Salman, J Khalid, M Abubakar (2018). Risk assessment of transgenic cotton against non-target whiteflies, thrips, jassids and aphids under field conditions in Pakistan. Intl J Curr Microbiol Appl Sci 6:11-24 
Ali I, NU Khan, S Gul, SU Khan, Z Bibi, K Aslam, G Shabir, HA Haq, SA Khan, I Hussain (2019a). Genetic Diversity and Population Structure Analysis in Upland Cotton Germplasm. Intl J Agric Biol 22:669-676

Ali MA, J Farooq, A Batool, A Zahoor, F Azeem, A Mahmood, K Jabran (2019b). Cotton Production in Pakistan. In: Cotton Production, 1st Edition, p:249. Jabran K, BS Chauhan (Eds.). John Wiley \& Sons Ltd., New York, USA

Allen G, M Flores-Vergara, S Krasynanski, S Kumar, W Thompson (2006). A modified protocol for rapid DNA isolation from plant tissues using cetyltrimethylammonium bromide. Nat Protoc 1:2320-2325

Ashraf S, AH Sangi, ZY Hassan, M Luqman (2018). Future of cotton sector in Pakistan: A 2025 outlook. Pak J Agric Res 31:145-150

Badigannavar A, GO Myers, DC Jones (2012). Molecular diversity revealed by AFLP markers in upland cotton genotypes. J Crop Improv 26:627-640

Becelaere GV, EL Lubbers, AH Paterson, PW Chee (2005). Pedigree-vs. DNA marker-based genetic similarity estimates in cotton. Crop Sci 45:2281-2287

Bertini CH, I Schuster, T Sediyama, EGD Barros, MA Moreira (2006). Characterization and genetic diversity analysis of cotton cultivars using microsatellites. Genet Mol Biol 29:321-329

Caetano-Anolles G (1997). Resolving DNA amplification products using polyacrylamide gel electrophoresis and silver staining. In Fingerprinting Methods Based on Arbitrarily Primed PCR pp:119-134. Springer, The Netherlands

Evanno G, S Regnaut, J Goudet (2005). Detecting the number of clusters of individuals using the software STRUCTURE: A simulation study. Mol Ecol 14:2611-2620

Gurmessa D (2019). Genetic diversity study of improved cotton ( $G$. hirsutum L.) varieties in Ethiopia using simple sequence repeats markers. J Biotechnol 7:6-14

Iqbal M, S Ul-Allah, M Naeem, M Ijaz, A Sattar, A Sher (2017). Response of cotton genotypes to water and heat stress: From field to genes. Euphytica 213:131

Jamil S, R Shahzad, SU Rahman, MZ Iqbal, M Yaseen, S Ahmad, R Fatima (2021). The level of Cry1Ac endotoxin and its efficacy against $H$. armigera in Bt. cotton at large scale in Pakistan. GM Crops Food 12:1-7

Jamil S, R Shahzad, S Kanwal, E Yasmeen, SU Rahman, MZ Iqbal (2020). DNA Fingerprinting and Population Structure of Date Palm Varieties Grown in Punjab Pakistan using Simple Sequence Repeat Markers. Intl J Agric Biol 23:943-950

Jans Y, WV Bloh, S Schaphoff, C Müller (2020). Global cotton production under climate change-Implications for yield and water consumption. In: Hydrology an Earth System Sciences Discussions, pp:1-27. https://doi.org/10.5194/hess-2019-595

Javaid A, F Awan, F Azhar, I Khan (2017). Assessment of allelic diversity among drought-resistant cotton genotypes using microsatellite markers. Genet Mol Res 16; Article gmr16029664

Kalia RK, MK Rai, S Kalia, R Singh, A Dhawan (2011). Microsatellite markers: An overview of the recent progress in plants. Euphytica 177:309-334

Khan AA, I Ashraf, G Hassan, S Ashraf (2016). On Farm Analysis of Cotton Growers Handicaps: Evidence from Cotton Belt of Pakistan. Intl J Agric Ext 4:79-85

Khan AI, FS Awan, B Sadia, RM Rana, IA Khan (2010). Genetic diversity studies among coloured cotton genotypes by using RAPD markers. Pak J Bot 42:71-77

Khanzada MS, TS Syed, S Rani, GHA Khanzada, M Salman, S Anwar, M Sarwar, AA Perzada, S Wang, AH Abro (2016). Occurrence and abundance of thrips, whitefly and their natural enemy, Geocoris Spp. on cotton crop at various localities of Sindh, Pakistan. J Entomol Zool Stud 4:509-515

Király I, R Redeczki, É Erdélyi, T Magdolna (2012). Morphological and molecular (SSR) analysis of old apple cultivars. Not Bot Hortic Agrobot Cluj-Nap 40:269-275

Lacape JM, D Dessauw, M Rajab, JL Noyer, B Hau (2007). Microsatellite diversity in tetraploid Gossypium germplasm: Assembling a highly informative genotyping set of cotton SSRs. Mol Breed 19:45-58

Lalwani S (2020). Pakistan in 2019: Navigating Major Power Relations amid Economic Crisis. Asian Survey 60:177-188
McCarty JC, DD Deng, JN Jenkins, L Geng (2018). Genetic diversity of day-neutral converted landrace Gossypium hirsutum L. accessions. Euphytica 214:1-14

Mumtaz AS, M Naveed, ZK Shinwari (2010). Assessment of genetic diversity and germination pattern in selected cotton genotypes of Pakistan. Pak J Bot 42:3949-3956

Nguyen TB, M Giband, P Brottier, AM Risterucci, JM Lacape (2004). Wide coverage of the tetraploid cotton genome using newly developed microsatellite markers. Theor Appl Genet 109:167-175

Noormohammadi Z, N Ibrahim-Khalili, M Sheidai, O Alishah (2018). Genetic fingerprinting of diploid and tetraploid cotton cultivars by retrotransposon-based markers. Nucleus 61:137-143

Noormohammadi Z, YF Hasheminejad-Ahangarani, M Sheidai, S Ghasemzadeh-Baraki, O Alishah (2013). Genetic diversity analysis in Opal cotton hybrids based on SSR, ISSR, and RAPD markers. Genetics and molecular research: Genet Mol Res 12:256-269

Pourabed E, MR Jazayeri Noushabadi, SH Jamali, N Moheb Alipour, A Zareyan, L Sadeghi (2015). Identification and DUS testing of rice varieties through microsatellite markers. Intl J Plant Genomics 2015; Article 965073

Pritchard JK, M Stephens, P Donnelly (2000). Inference of population structure using multilocus genotype data. Genetics 155:945-959

Rana AW, A Ejaz, SH Shikoh (2020). Cotton crop: A situational analysis of Pakistan. In: Prepared as part of the technical assistance to ministry of national food security and research. International Food Policy Research Institute, Government of Pakistan.

Rehman A, L Jingdong, AA Chandio, I Hussain, SA Wagan, QUA Memon (2019). Economic perspectives of cotton crop in Pakistan: A time series analysis (1970-2015)(Part 1). J Saud Soc Agric Sci 18:49-54

Rohlf F (1998). NTSYS-pc version 2.0. Numerical Taxonomy and Multivariate Analysis System, pp:1-43. Exeter software, Setauket, New York

Santhy V, M Meshram, H Santosh, K Kranthi (2019). Molecular diversity analysis and DNA fingerprinting of cotton varieties of India. Ind $J$ Genet Plant Breed 79:719-725

Shah ZH, M Munir, AM Kazi, T Mujtaba, Z Ahmed (2009). Molecular markers based identification of diversity for drought tolerance in bread wheat varieties and synthetic hexaploids. Curr Issues Mol Biol 11:101-110

Sheidai M, F Afshar, M Keshavarzi, SM Talebi, Z Noormohammadi, T Shafaf (2014). Genetic diversity and genome size variability in Linum austriacum (Lineaceae) populations. Biochem Syst Ecol 57:20-26

Shuli F, AH Jarwar, X Wang, L Wang, Q Ma (2018). Overview of the cotton in Pakistan and its future prospects. Pak $J$ Agric Res 31:396-408

Singh S (2017). "White Gold" for Whom? A Study of Institutional Aspects of Work and Wages in Cotton GPNs in India. In: Critical Perspectives on Work and Employment in Globalizing India pp:15-36. Springer, The Netherlands

Townsend T (2020). World natural fibre production and employment. In: Handbook of Natural Fibres, pp: 15-36. Elsevier, Amsterdam, The Netherlands

Ul-Allah S, S Ahmad, M Iqbal, M Naeem, M Ijaz, M Qadir, ZH Ahmad, HG Nabi (2019). Creation of new genetic diversity in cotton germplasm through chemically induced mutation. Intl J Agric Biol 22:51-56

Ullah I, A Iram, M Iqbal, M Nawaz, S Hasni, S Jamil (2012). Genetic diversity analysis of $\mathrm{Bt}$ cotton genotypes in Pakistan using simple sequence repeat markers. Genet Mol Res 11:597-605

UPOV-BMT B (2002). 36/10 Progress report of the $36^{\text {th }}$ session of the technical committee, the technical working parties and working group on biochemical and molecular techniques and DNA-profiling in particular. Upov-Bmt, Geneva, Switzerland

Yu J, S Jung, CH Cheng, SP Ficklin, T Lee, P Zheng, D Jones, RG Percy, D Main (2014). CottonGen: A genomics, genetics and breeding database for cotton research. Nucl Acids Res 42:1229-1236

Zhang Y, M Kuang, W Yang, H Xu, D Zhou, Y Wang, X Feng, C Su, F Wang (2013). Construction of a primary DNA fingerprint database for cotton cultivars. Genet Mol Res 12:1897-1906

Zhu L, P Tyagi, B Kaur, V Kuraparthy (2019). Genetic diversity and population structure in elite US and race stock accessions of upland cotton (Gossypium hirsutum). J Cotton Sci 23:38-47 